\title{
Food and feed sampling: balancing ethics and money Claudia Paoletti
}

Program Manager, Transformation Unit - TS, European Food Safety Authority EFSA, Via Carlo Magno 1/A, 43100 Parma, Italy

I first accepted the editor's invitation to contribute to this special issue on "economic arguments for representative sampling" with great enthusiasm. Alas, a few hours later, the enthusiasm started to fade because the many experiences of resistance to putting the Theory of Sampling (TOS) into practice in the food and feed sector came back to me. However, upon considerable soul searching, there may still surely be hope!

\section{A personal statement}

I have devoted about 20 years of my professional career to studying and regulating food and feed sampling standards and normative documents. ${ }^{1-8}$ The good news is that many of them (though not all) claim that sampling should be representative. The bad news is that almost none goes as far as claiming representativeness as a mandatory requirement, the only exceptions being DS 3077, ${ }^{9}$ Recommendation EC $787^{10}$ (2004) and prEN ISO - 21568 (2005). The unavoidable result is that these standards fail when applied in practice, creating a breach between the principles behind the TOS's goals (good) and its application to everyday reality in the food and feed arena (bad). Thus, sampling is often felt as a necessity to be fulfilled to collect material for analytical investigation-clearly not knowing or reflecting on how important this information is for making societal decisions about public nutrition and health. However, reducing to a minimum the time devoted to sampling ("the faster the better") and minimising the associated

\section{DOI: $10.1255 /$ sew.2021.a47}

(C) 2021 The Author

Published under a Creative Commons BY-NC-ND licence

\section{(c) $(\Theta \Theta \Theta$}

costs ("the cheaper the better") will sooner or later sacrifice sampling quality and reliability. As for everything else in life, quality does not go together with speed and lack of resources.

\section{Setting a constructive scene}

Since the present focus is on economic arguments for doing the right thing, instead of repeating that non-representative sampling is useless by definition, and that every penny spent on collecting specimens and analysing them is wasted, I would rather tackle the issue from the other end, exploring what happens when "something wrong" is detected in a food or feed product.

\section{Looking rationally at the costs involved}

When a food or a feed product turns out to be non-compliant with a priori established quality/safety criteria, the product needs to be removed from the market. What are the costs of removal? Per product the overall financial losses include all production, distribution and selling costs already sustained before the decision to pull from the market. Plus the costs necessary to i) map the supply-distribution followed to place the product on the market; ii) removal of the product from every supermarket counter and storage room across all the regions, countries and possibly continents to which the product was distributed; iii) costs to destroy the product. Arguably, these total costs are much, much higher than the cost required for the a priori application of a TOS-compliant sampling method, allowing the analysis of representative samples to support well-substantiated and informed decisions before market release.

When we total up the costs for this, grave problems become evident. Because of the vast amounts and tonnages involved, the costs are in fact

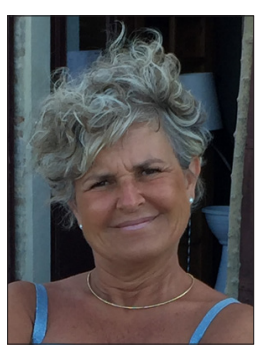

so massive that they cannot even be estimated with reasonable precision, but they are guaranteed to be huge.

\section{Scientific and technological} understanding does not hurt Under a less catastrophic scenario, a reliable understanding of human and animal exposure to certain substances (e.g. pesticides) is an important and wise requirement under many jurisdictions. The earlier it is understood that only representative samples reduce the possibilities of either mis-estimating actual exposure levels for humans and animals or, worse, under-estimating the risks for consumers to exceed tolerable intake levels, the better for society. This is also important in the case of foods and feed with nutritional benefits, where under- or over-estimating intake levels may lead to nutritional or deficiency problems. This also plays a critical role regarding surveillance of foods and feeds with unintentional contaminants or intentional adulterations, due to their often-low concentration levels and highly heterogeneous distributions. Watching out for these societal risks ranks among the prime objectives of national and international regulating authorities charged with consumer safety. These are goals worthy our most ardent efforts. But are we doing well enough?

Reality check: very different objectives and usages of the TOS

Well, in today's food and feed arena, sampling continues to be perceived more as an economic burden and a technical necessity to be fulfilled because of regulatory demands, rather than a need to ensure proper citizen and/or animal protection.

Also, readers of this column could well be staggered, and maybe confused, 
by the completely different attitude towards sampling between, for example, the mining/minerals/cement and the food \& feed industry sectors. In the world of geological resource-based businesses, incorrect sampling means huge economic losses as value, cost and profitability estimates can be made precisely because the TOS is available. Here the TOS can be seen as the operative element safeguarding the business endeavours, see examples from the wide history of TOS applications, well substantiated in the annals of the world sampling community. Whereas in the food and feed business, sampling is a scientific tool to verify the accuracy of specific product claims, or to search for possible contaminants, or toxins, allergens, pollutants etc. Here, in essence, sampling means searching for possible problems, or verifying their absence to a certain degree of confidence (the concept of "risk assessment").

This contra-positioning is a key point for samplers, process engineers, managers, regulators, investors: IF from a practical point of view, exploration and searching for metalliferous resources and ores ${ }^{\mathrm{a}}$ is not so different from searching for, e.g., aflatoxins in a 60,000-ton shipment of grain kernels, or searching for accidental manufacturing residues across millions of chocolate bars (or a thousand barrels of pet food)-in practice the motivations for investing in correct sampling are markedly different. In the mining/minerals sectors the better the sampling the better for business (better in a straight economic optimisation sense), whereas in the food/feed sectors the better the sampling, the higher the risk of lot rejection or similar, which always carries a heavy negative economic penalty. What is good for one type of business is bad for another-what is good for one type of societal enterprise, is bad for others-the

aplease don't just think of gold or diamonds, which geologically are kind of atypical resources-distinguishing themselves only by the societal agreement that they represent great value. The value of the much more voluminous base metals a.o. commodities, is vastly greater.

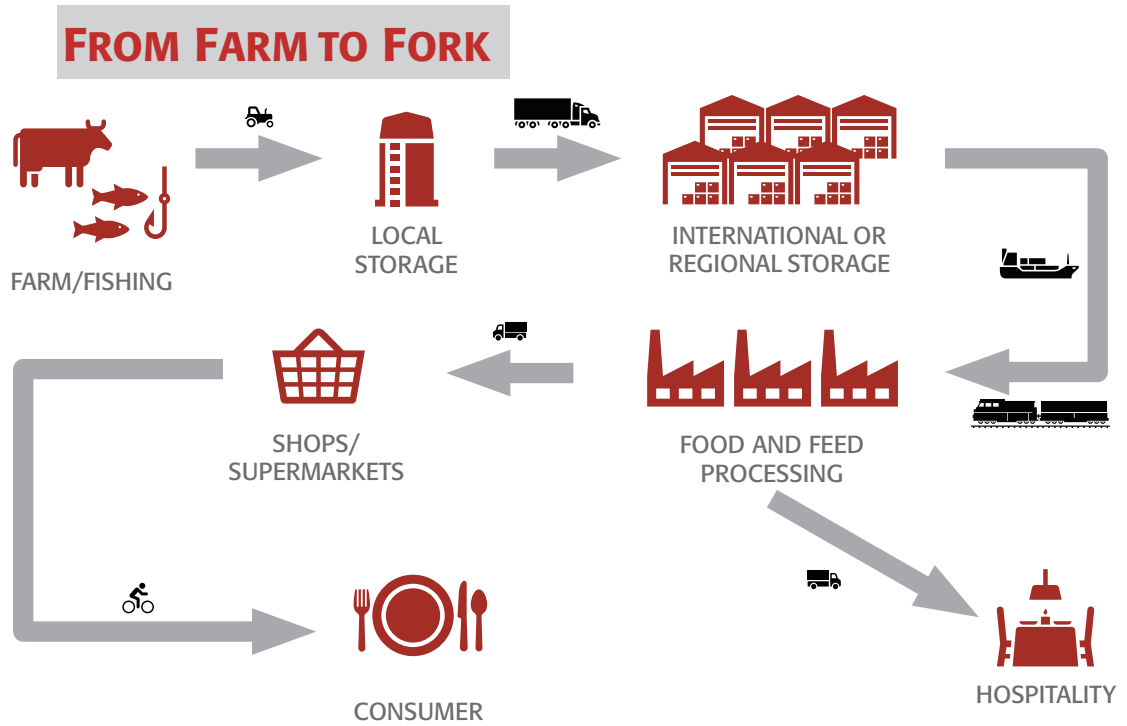

The food supply/production pathway: "From field to fork".

gamut of TOS applications in the last 20 years documents this dichotomy.

\section{Balancing the opposites}

The need for balance between integrity and financial gain opens up a quite different discussion on a higher level: one about direct use and benefits vs indirect and intangible disadvantages of the TOS involvement, which in the main goes beyond the purpose of the specific topic of this column, but here is at least the gist of it.

When sampling is executed to check for compliance with legislation requirements (i.e. regulatory sampling) it should be of crucial importance to ensure a high degree of confidence that the survey is accurate (unbiased) and that the compound sampling error is as small as indeed possible, within specified economic and workload boundaries. Specifically, if there is a legal threshold limit set for acceptance of the presence of a specific substance, all adopted sampling protocols must ensure that such threshold is respected with the specified degree of confidence. Of course, the lower this limit is, the greater the demands will be upon the sampling procedures and plans-and this cannot avoid being associated with some added costs.

Europe has established a very stringent approach to food and feed safety, monitoring products throughout all the steps of their production chain, "from farm to fork". Embedded into such a solid and ambitious safety strategy, and almost always out of sight, there is a high demand for accurate and precise, i.e. representative, sampling procedures, capable of ensuring reliable estimations throughout this entire pathway, leaving very little space for shortcuts behind the cheap and fast collection of meaningless (i.e. non-representative) specimens.

\section{Where does this leave us- Trust!}

In the food and feed sector, however you look at sampling, it is never only about money: it is about ethics and money. Correct sampling is not a money maker as in other sectors. Appropriate sampling is about being accountable for the trust that society puts into governmental and inter-governmental control systems for the safety of food and feed products. Society has no other choice!

After reading this article, you will sooner or later open the refrigerator and eat food that you bought at a supermarket. You trust it as safe. You trust that the control system worked to protect you. Consciously or unconsciously you trust the sampling adopted by such a control system was appropriate, i.e. representative, meaning that the safety decision taken applies also to the portion you 
have in your refrigerator. If you again think of the dimensions of the global market, this is extremely far from being a trivial personal issue-the job to ensure for food and feed safety for all consumers is enormous! Ultimately, the money invested for correct sampling is money invested for the citizens who have neither the means, nor the knowledge, to verify. This trust should have much more exposure within and especially beyond our scientifically and technically driven community. This trust should become the root reason to ensure a continuous and open dialogue between TOS experts and those who decide what ultimately is allowed on the market: the consumers eat what reaches supermarket shelves.

After 20 years-my last effort? Allow me to borrow DrVogel's statement (elsewhere in this column): "If 'representative' is removed from the sampling process, all 'piece of mind' goes away!".

The worst situation is that as long as nobody finds problems, everybody lives happily. Alas, everybody lives, but blindly! Are we ready to deal with these topics-going beyond profitability-transparently and honestly? Until now this would not appear to have been greatly successful.

Hopefully, the future debate will fuel more active measures, including reactions to this multi-authored contribution, surprising us!

\section{Disclaimer}

The author declares no competing interest. Claudia Paoletti is employed by the
European Food Safety Authority (EFSA). The positions and opinions presented in this article are those of the author alone and do not necessarily represent the views of scientific works of EFSA.

\section{References}

1. K.H. Esbensen and C. Paoletti, "Theory of sampling (TOS): pro et contra", Spectrosc. Europe 30(1), 23-26 (2018). https://www.spectroscopyeurope.com/sampling/ theory-sampling-tos-pro-et-contra

2. C. Paoletti and K.H. Esbensen, "Distributional assumptions in food and feed commodities-development of fit-for-purpose sampling protocols", J. AOAC Int. 98(2), 295-300 (2015). https://doi. org/10.5740/jaoacint.14-250

3. H.A. Kuiper and C. Paoletti, "Food and feed safety assessment: the importance of proper sampling", J. AOAC Int. 98(2), 252-258 (2015). https:// doi.org/10.5740/jaoacint.15-007

4. K.H. Esbensen, C. Paoletti and P. Minkkinen, "Representative sampling of large kernel lots I. Theory of Sampling and variographic analysis", Trends Anal. Chem. 32, 154-164 (2012). https://doi.org/10.1016/j. trac.2011.09.008

5. P. Minkkinen, K.H. Esbensen and C. Paoletti, "Representative sampling of large kernel lots II. Application to soybean sampling for GMO control", Trends Anal. Chem. 32, 165-177 (2012). https://doi.org/10.1016/j. trac.2011.12.001

6. K.H. Esbensen, C. Paoletti and P. Minkkinen, "Representative sampling of large kernel lots III. General considerations on sampling heterogeneous foods", Trends Anal. Chem. 32, 178-184 (2012). https://doi. org/10.1016/j.trac.2011.12.002

7. C. Paoletti, A. Heissenberger, M. Mazzara, S. Larcher, E. Grazioli, P. Corbisier, N. Hess, G. Berben, P.S. Lübeck, M. De Loose, G. Moran, C. Henry, C. Brera, I. Folch, J. Ovesna and G. Van den Eede, "Kernel lot distribution assessment (KeLDA): a study on the distribution of GMO in large soybean shipments", Eur. Food Res. Technol. 224, 129-139 (2006). https://doi.org/10.1007/s00217006-0299-8

8. C. Paoletti, M. Donatelli, S. Kay and G. Van den Eede, "Simulating kernel lot sampling: the effect of heterogeneity on the detection of GMO contaminations", Seed Sci. Technol. 31 (3), 629-638 (2003). https://doi. org/10.15258/sst.2003.31.3.12

9. DS 3077. Representative Sampling - Horizontal Standard. Danish Standards (2013). https://www.ds.dk

10. Commission Recommendation of 4 October 2004 on Technical Guidance for Sampling and Detection of Genetically Modified Organisms and Material Produced from Genetically Modified Organisms as or in Products in the Context of Regulation (EC) No 1830/2003 (2004). https://op.europa.eu/en/publicationdetail/-/publication/e2eec03b-fb4c4b99-a 114-bbb257aace97 